\title{
QUANTIFICAÇÃO DO STONE CLINIC EFFECT EM PACIENTES COM NEFROLITÍASE
}

\author{
Maurício Carvalho*, Ana Claudia H. Ferrari, luciana O. Renner, Marcos A. Vieira, Miguel Carlos Riella \\ Trabalho realizado no Ambulatório de Pesquisa em Nefrolitíase do Hospital Universitário Evangélico e \\ Instituto do Rim - Ambulatório de Pesquisa em Nefrolitíase, Curitiba, PR.
}

RESUMO - 0 termo stone clinic effect refere-se ao efeito do aumento da ingestão hídrica e aconselhamento dietético na evolução clínica da doença calculosa renal.

OBJEtivo. 0 nosso objetivo foi quantificar esta variável em pacientes portadores de nefrolitíase.

Métodos. Vinte e cinco pacientes (II mulheres e 14 homens; $47,64 \pm 10,55$ anos) com nefrolitíase recorrente foram acompanhados com consultas trimestrais por um ano. Orientações sobre aumento da ingestão hídrica, diminuição do consumo de sal e proteína foram rotineiramente fornecidas. Nenhum paciente foi submetido à terapia farmacológica. Foram coletados no início (SI) e no final do seguimento (S2) os seguintes parâmetros: atividade clínica e radiológica da doença litiásica, urina de 24 horas com dosagem de creatinina, cálcio, sódio, ácido úrico, citrato, oxalato e magnésio. A supersaturação (SS) para o oxalato de cálcio foi calculada pelo índice de Tiselius.
Resultados. Onze (44\%) pacientes apresentaram hipocitratúria, nove (36\%) hipercalciúria e cinco $(20 \%)$ hiperuricosúria. Houve aumento do volume urinário $(1903 \pm 8 \mathrm{II}$ vs $238 \mathrm{I} \pm 919 \mathrm{ml} /$ dia, $p<0,05)$ e diminuição significativa na SS urinária $(I, I 3 \pm 0,8$ vs $0,6 \pm 0,2, p<0,0 I)$. 0 número de cálculos formados foi menor quando comparado com o mesmo período de seguimento pré-estudo $(S \mid=2,04 \pm 1,51$ vs $S 2=0,72 \pm 0,23$ cálculos, $p<0,00 I)$. Não houve formação ou crescimento de cálculos preexistentes em 20 dos 25 pacientes (80\%).

Conclusõos. $\mathbf{O}$ stone clinic effect diminuiu significativamente a SS urinária para 0 oxalato de cálcio e a ocorrência de novos cálculos em $80 \%$ dos pacientes durante o primeiro ano de acompanhamento.

Unitermos: Litíase renal. Calculose renal. Distúrbios metabólicos. Cálcio.

\section{INTRODUÇÃO}

A formação de cálculos renais requer a produção de fases sólidas na urina, como as de oxalato de cálcio, fosfato de cálcio, ácido úrico e outras menos comuns. A força motriz para a cristalização intratubular renal é denominada de supersaturação (SS) e pode ser expressa como a relação entre a concentração de um determinado sal em solução dividido pela solubilidade deste mesmo sal na urina a $37^{\circ} \mathrm{C}$. Aumentando-se progressivamente a SS, um ponto é atingido no qual a cristalização tem início. Este ponto é denominado de limite superior da metaestabilidade.

Vários trabalhos demonstraram correspondência entre SS urinária e composição dos cálculos formados ${ }^{2}$. Além disto, medidas para diminuir a SS urinária

* Correspondência:

Instituto do Rim - Ambulatório de Pesquisa em Nefrolitíase.

Rua Augusto Stelfeld, 2034 - Cep: 80730-150

Curitiba - PR - Tel.: (41) 322-0509

E-mail:carvalho@mais.sul.com.br diminuem a ocorrência de novos cálculos ${ }^{3}$. Estudos observacionais e controlados verificaram que a ingestão hídrica reduzida, o aumento do consumo de proteínas e a diminuição dietética de cálcio são fatores de risco para a formação de cálculos renais ${ }^{4,5,6}$. O decréscimo na ingestão de líquidos reduz o volume urinário, aumenta a concentração relativa dos principais sais envolvidos na litogênese e conseqüentemente eleva a SS.

O chamado efeito do ambulatório especializado em nefrolitíase (stone clinic effect) refere-se à eficiência do aumento da ingestão hídrica e aconselhamento dietético, comumente oferecido aos pacientes, na evolução clínica da doença litiásica. Em um estudo pioneiro, foi observada redução de 58\% na formação e crescimento de cálculos renais após a adoção sistemática destas medidas? No nosso meio, peculiaridades dietéticas, como a baixa ingestão de cálcio, tornam difícil a extrapolação de resultados obtidos em outros países ${ }^{8}$. O objetivo deste estudo é quantificar o stone clinic effect em portadores de nefrolitíase.

\section{Métodos}

Foram recrutados 25 pacientes, portadores de nefrolitíase recorrente, em acompanhamento no Ambulatório de Pesquisa em Nefrolitíase do Hospital Evangélico de Curitiba, Paraná. A seleção foi feita de modo consecutivo, em um período de 30 dias, entre fevereiro e março de 200I. A presença de distúrbios do metabolismo do cálcio como hiperparatiroidismo primário, rim de esponja medular, acidose tubular renal, malignidade e déficit de função renal (depuração de creatinina menor que $60 \mathrm{ml} / \mathrm{min}$ ) constituíram critérios de exclusão.

Todos os pacientes foram submetidos à consulta médica no início do acompanhamento e a cada três meses posteriormente, durante o período de um ano. Orientações sobre aumento da ingestão hídrica (manter volume urinário maior que dois litros ao dia), diminuição do consumo de sal (menor que $200 \mathrm{mEq} / \mathrm{dia}$ ) e proteína (em torno de $\mathrm{lg} / \mathrm{kg} / \mathrm{dia}$ ) e ingestão adequada de cálcio (800 mg/dia) foram rotineiramente fornecidas pela equipe médica. Nenhum paciente foi submetido à terapia farmacológica específica, exceto antiespasmódicos e/ou antiinflamatórios para controle da dor. 
Na primeira consulta estimava-se a atividade clínica da doença litiásica no ano imediatamente anterior ao estudo, avaliando-se número de crises, episódios de hematúria ou infecções do trato urinário, eliminação de cálculos e realização de procedimentos urológicos, como litotripsia extracorpórea, por exemplo. Este mesmo procedimento foi adotado após cada consulta trimestral até o final do estudo. Foi possível obter de todos os pacientes estudados pelo menos um estudo de imagem realizado previamente ao início do estudo.

No início (SI) e no final do seguimento (S2) todos os pacientes foram submetidos à avaliação radiológica e metabólica. Urografia excretora, raios $X$ simples de abdome e ultrasonografia foram realizados de acordo com a conveniência do serviço. A recorrência foi julgada pelo aparecimento de novos cálculos ou por aumento superior a 50\% no diâmetro dos preexistentes.

A avaliação metabólica consistiu de duas amostras de urina de 24 horas, colhidas com intervalo de pelo menos 30 dias após episódio de cólica nefrética, com os pacientes em dieta habitual, analisando-se os seguintes parâmetros: creatinina, cálcio, sódio, ácido úrico, citrato, oxalato e magnésio. A creatinina foi medida pelo método do picrato alcalino, segundo a reação de Jaffé, com valores de referência de 1000 a 2000 mg/dia para homens e 800 a 1500 mg/dia para mulheres. O cálcio foi medido por método enzimático colorimétrico, considerado como valor normal menor que $4 \mathrm{mg} / \mathrm{kg}$ de peso/dia para ambos os sexos. O sódio foi medido por espectrofotometria de chama, com valores normais de 50 a $150 \mathrm{mEq} / \mathrm{dia}$. O ácido úrico urinário foi analisado pelo método da uricase, com valores considerados normais abaixo de $750 \mathrm{mg} / \mathrm{dia}$. O citrato foi medido pela enzima citrato-liase, com valores aceitos como normais acima de $320 \mathrm{mg} / \mathrm{dia}$. O oxalato foi determinado pela técnica da enzima oxalato oxidase, com limite de normalidade de $45 \mathrm{mg} /$ dia. O magnésio foi avaliado por método enzimático colorimétrico, com valores considerados dentro da faixa de referência entre 50 a $150 \mathrm{mg} / \mathrm{dia}$. Também foram determinados o $\mathrm{pH}$ e o volume urinário. Para cada conjunto de duas amostras, foi utilizada a média dos valores obtidos. A SS urinária para o oxalato de cálcio foi calculada pelo

\begin{tabular}{|c|c|c|c|c|c|c|c|}
\hline \multicolumn{8}{|c|}{ Tabela I - Bioquímica urinária } \\
\hline & Volume & Citrato & Cálcio & Ac.úrico & Oxalato & Sódio & Magnésio \\
\hline $\begin{array}{l}\text { Início (SI) } \\
\text { Final (S2) }\end{array}$ & $\begin{array}{c}|903 \pm 81| \\
2381 \pm 9 \mid 9 *\end{array}$ & $\begin{array}{c}368 \pm 238 \\
502 \pm 221 *\end{array}$ & $\begin{array}{l}202 \pm 108 \\
193 \pm 126\end{array}$ & $\begin{array}{c}514 \pm 226 \\
395 \pm 172 *\end{array}$ & $\begin{array}{c}29,5 \pm 13,2 \\
25,7 \pm 9,7\end{array}$ & $\begin{array}{l}223 \pm 96,6 \\
|8| \pm 71,2\end{array}$ & $\begin{array}{c}60,5 \pm 18 \\
88 \pm 15\end{array}$ \\
\hline
\end{tabular}

*p $<0,05$ quando comparado com o início (SI)

\begin{tabular}{|c|c|c|c|}
\hline \multicolumn{4}{|c|}{ Tabela 2 -Anormalidades metabólicas (urina 24 horas) } \\
\hline & Número de pacientes & Início(SI) & Final (S2) \\
\hline $\begin{array}{l}\text { Hipocitratúria } \\
\text { (Citrato mg/dia) }\end{array}$ & 11 & $|78| \pm 93,3$, & $349 \pm|4| *$ \\
\hline $\begin{array}{l}\text { Hipercalciúria } \\
\text { (Cálcio mg/dia) }\end{array}$ & 9 & $323 \pm 62$ & $234 \pm 114$ \\
\hline $\begin{array}{l}\text { Hiperuricosúria } \\
\text { (Ac.úrico mg/dia) }\end{array}$ & 5 & $866,5 \pm 48,6$ & $609 \pm 165$ \\
\hline $\begin{array}{l}\text { Baixo volume } \\
\text { (Diurese ml/dia) }\end{array}$ & 5 & $975 \pm 195$ & $1674 \pm 630$ \\
\hline $\begin{array}{l}\text { Hiperoxalúria } \\
\text { (Oxalato mg/dia) }\end{array}$ & 3 & $48,33 \pm 3,21$ & $33,3 \pm 16,2$ \\
\hline
\end{tabular}

índice de Tiselius ${ }^{9}$, de acordo com a seguinte fórmula: $\operatorname{AP}(\mathrm{CaOx})=1,9 \times \mathrm{Cálcio}^{0,84} \times$ Oxalato $\times$ Citrato ${ }^{-0,22} \times$ Magnésio $^{-0,12} \times$ Volume $^{-1,03}$.

Os dados são apresentados como média \pm desvio padrão. Para análise dos resultados foi utilizado o teste $t$ de Student para amostras independentes. A freqüência de eventos clínicos e de novos cálculos foi comparada pela técnica do $X^{2}$. Os achados foram considerados significantes quando $p<0,05$.

\section{Resultados}

O estudo incluiu 14 homens e II mulheres, com idades de $50,9 \pm 11$ e $43,4 \pm 8,6$ anos de idade, respectivamente $(p<0,07)$. Foi detectada pelo menos uma alteração metabólica em todos os pacientes estudados. $\mathrm{Na}$ avaliação inicial (SI) II pacientes (44\%, seis homens e cinco mulheres) apresentaram-se com hipocitratúria, com excreção urinária de $178 \pm 93,3 \mathrm{mg} /$ dia, nove pacientes (36\%, seis homens e três mulheres) com hipercalciúria de $323 \pm 62 \mathrm{mg} / \mathrm{dia}$, cinco (20\%, dois homens e três mulheres) com hiperuricosúria, com excreção média de 866 $\pm 49 \mathrm{mg} /$ dia de ácido úrico e cinco com baixo volume urinário $(20 \%$, três homens e duas mulheres, diurese média de $975 \pm 195 \mathrm{ml} /$ dia). Três pacientes apresentaram ainda hiperoxalúria ( $12 \%$, dois homens e uma mulher, excreção média de 48,3 $\pm 3,21 \mathrm{mg} / \mathrm{dia}$ ).
Ao final do acompanhamento (\$2), ao analisar-se todos os pacientes como um único grupo, houve aumento significativo do volume urinário (1903 \pm 81 I vs $2381 \pm 919 \mathrm{ml} / \mathrm{dia}$, $p<0,05)$ e da excreção de citrato $(368 \pm 238$ vs. $502 \pm 221 \mathrm{mg} / \mathrm{dia}, \mathrm{p}<0,05)$, assim como diminuição da uricosúria (5/4 4226 vs. $395 \pm$ $172 \mathrm{mg} / \mathrm{dia}, \mathrm{p}<0,05)$. Ocorreu também queda na calciúria, na oxalúria e na excreção de sódio, porém de maneira não significativa (Tabela I). Notou-se também, em cada grupo de pacientes com anormalidades específicas relacionadas à litogênese, uma melhora do padrão de excreção urinária (Tabela 2). Como conseqüência, houve decréscimo na SS urinária para o oxalato de cálcio $(1,13 \pm 0,8$ vs $0,6 \pm 0,2, p<0,01)$.

No ano prévio ao início do seguimento (SI), 23 dos 25 pacientes relataram 32 crises de dor, eliminação de sete cálculos e haviam realizado 18 procedimentos urológicos, a maioria deles litotripsia extracorpórea. Durante o período de estudo, 12 pacientes referiram 15 episódios de cólica nefrética ( $p<0,05$ comparado com o ano anterior ao acompanhamento), sete cálculos foram eliminados e 10 sessões de litotripsia foram realizadas em sete pacientes.

O número de cálculos formados por paciente foi menor quando comparado com a avaliação pré-estudo $(S|=2,04 \pm|, 5 \mid$ vs 


\section{Figura I - Stone clinic effect e recorrência em nofrolitíase}

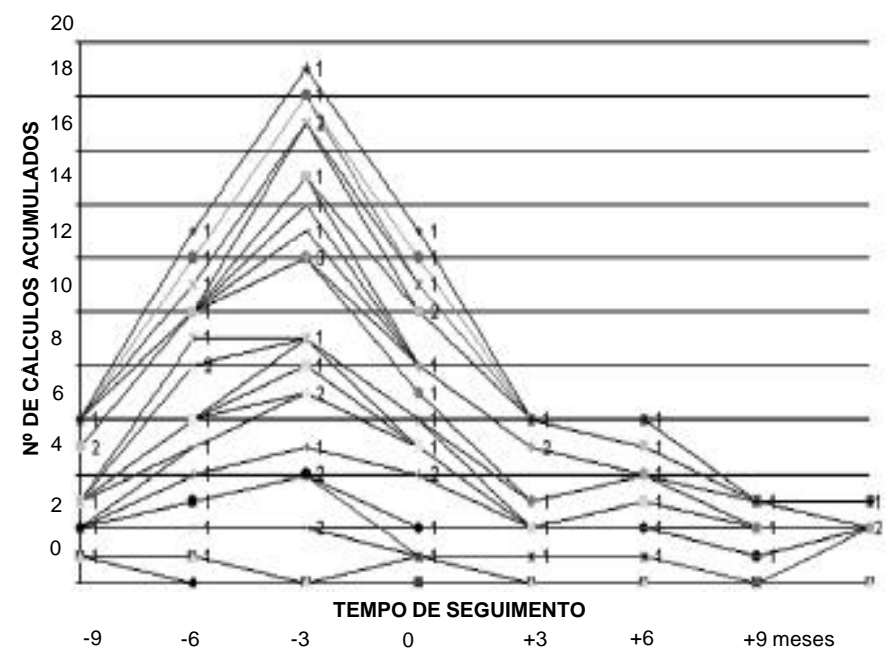

$S 2=0,72 \pm 0,23$ cálculos, $p<0,00 I$ ). Não houve formação ou crescimento de cálculos preexistentes em 20 dos 25 pacientes $(80 \%, p<0,00$ I) (Figura I).

\section{Discussão}

A eficiência do tratamento médico na nefrolitíase pode ser medida por redução na taxa de SS urinária e por diminuição na excreção de vários elementos ditos litogênicos presentes na urina, como por exemplo cálcio, oxalato e ácido úrico ${ }^{10}$. Entretanto, estudos que demonstram eficácia por meio de desfechos intermediários devem ser interpretados como promissores, e não como definitivos. Apesar de haver alguma correlação entre desfechos substitutos e relevantes, a associação entre eles pode não ser linear. Em algumas situações clínicas, eficácia em desfechos substitutos pode resultar em ausência de benefícios clinicamente significativos.

O nosso estudo demonstrou que o tratamento dietético, baseado no aumento da ingestão hídrica, redução do consumo de sal e de proteínas de origem animal, o chamado stone clinic effect, além de diminuir a SS urinária reduz eficazmente a formação de cálculos renais. De fato, houve formação de novos cálculos em apenas cinco (20\%) pacientes. Além disso, 12 pacientes referiram episódios de cólica nefrética contra 23 dos 25 pacientes no ano prévio ao início do seguimento $(p<0,05)$.
Em nosso meio, até $49 \%$ dos pacientes podem apresentar baixo volume urinário, principalmente em regiões de clima quente ${ }^{\prime \prime}$. Portanto, um grupo considerável de pacientes pode ser tratado apenas com aconselhamento dietético ${ }^{12}$. Uma das medidas mais simples e eficientes no tratamento da nefrolitíase é o aumento da ingestão hídrica. Recomenda-se que pelo menos $50 \%$ do volume ingerido seja de água. Com o aumento de consumo de água mineral, existe preocupação com a quantidade de eletrólitos presentes nestes compostos. Há uma grande variação no conteúdo mineral de águas disponíveis no comércio ${ }^{13}$. Este fato deve ser levado em consideração na seleção. Porém, o impacto clínico da dureza da água (definida como a soma das concentrações de cálcio e magnésio) na nefrolitíase permanece incerto, com a maioria dos estudos demonstrando apenas fracas correlações entre o índice de dureza e a excreção urinária de cálcio, magnésio e citrato $^{14}$. Maiores estudos, de caráter epidemiológico, são necessários para esclarecimento desta questão ${ }^{15}$.

Em pacientes hipercalciúricos, restrição moderada de proteínas diminui a excreção urinária de cálcio, provavelmente por uma redução na reabsorção óssea causada pela diminuição da carga de ácidos exógenos. Além disto, hiperuricosúria, hiperoxalúria e hipocitratúria estão freqüentemente associadas em pacientes com alta ingestão protéica' ${ }^{16}$.
Recentemente, Reddy e colaboradores concluíram que dietas com baixo teor de carboidratos e ricas em proteínas aumentam o risco de formação de cálculos renais, causam balanço negativo de cálcio e podem aumentar o risco de perda óssea ${ }^{17}$.

Além da diminuição da cristalização, valores reduzidos de SS urinária promovem um melhor funcionamento da proteína de TammHorsfall, inibindo mais eficientemente a agregação de partículas dentro do trato urinário ${ }^{18}$.

Este trabalho apresenta algumas limitações. O número de pacientes estudados foi pequeno. Porém, a amostra é representativa da população nefrolitiásica vista em nosso meio, os distúrbios metabólicos seguem o padrão de distribuição observado em outras publicações nacionais e a seleção foi feita de modo consecutivo, procurando-se apenas excluir os pacientes com hipercalcemia e déficit de função renal associado. Outro fator limitante é o pouco tempo de seguimento. Hosking e colaboradores, após um período de acompanhamento de 62,6 meses, observaram inatividade metabólica em 58,3\% dos pacientes ${ }^{7}$. Provavelmente, com um tempo maior de observação, o índice de $80 \%$ obtido em nosso estudo seria menor, considerando-se taxas de recorrência de até $20 \%$ ao ano em pacientes tratados apenas com intervenção dietética ${ }^{19}$.

\section{Conclusóes}

As intervenções dietéticas, denominadas coletivamente de stone clinic effect, diminuíram significativamente a SS urinária, o número de cálculos formados e $80 \%$ dos pacientes não apresentaram recorrência da doença litiásica no primeiro ano de acompanhamento.

Fontes de financiamento: Hospital Universitário Evangélico e Fundação Pró-Renal - Curitiba - PR.

\section{SUMMARY}

Quantification OF the STONe Clinic EFFECT IN PATIENTS WITH NEPHROLITHIASIS

BACKGROUND. The "stone clinic effect" refers to the effect of encouraging a high intake of fluid and diet counseling in the clinical evolution of kidney stone disease. OBJECTIVE: Our objective was to determine the extent of this variable in patients with nephrolithiasis. 
Methods. Twenty-five patients (II female and 14 male; $47.64 \pm 10.55$ years old) with recurrent nephrolithiasis were prospectively followed for one year, with 3-month interval medical evaluation. Patients were advised to increase the fluid intake, and to limit the intake of salt and protein. No patient was submitted to pharmacological therapy. Two 24-hour urine samples were collected at baseline (SI) and in the end of follow-up (\$2) for the measurement of creatinine, calcium, sodium, uric acid, citrate, oxalate and magnesium. Metabolic and radiological activity was also assessed. Urinary calcium oxalate supersaturation (SS) was calculated by Tiselius index.

Results. Eleven (44\%) patients had hypocitraturia, 9 (36\%) hypercalciuria and 5 (20\%) hyperuricosuria. Urinary volume increased (1903 \pm 811 vs. $2381 \pm 919 \mathrm{~m} / /$ day, $p<0.05$ ) and $S S$ decreased significantly (1.13 \pm 0.8 vs. $0.6 \pm 0.2, p<0.01)$. During follow-up, the number of stones decreased when compared to baseline $(S I=2.04 \pm 1.51$ vs. $S 2=$ $0.72 \pm 0.23$ stones, $p<0.001)$. There was no evidence of new stone formation or growth in 20 of 25 patients (80\%).

Conclusions. The "stone clinic effect" significantly decreased urinary SS for calcium oxalate and the formation of new kidney stones in $80 \%$ of patients during first year of follow-up. [Rev Assoc Med Bras 2004; 50(I): 79-82]

KEY wORDs: Nephrolithiasis. Renal stone. Metabolic alterations. Calcium.

\section{REFERÊNCIAS}

I. Finlayson B. Physicochemical aspects of urolithiasis. Kidney Int 1978; 13:344-60.

2. Parks JH, Coward M, Coe FL. Correspondence between stone composition and urine supersaturation in nephrolithiasis. Kidney Int 1997; 51:894-900.

3. Carvalho M, Nakagawa Y. Supersaturação urinária e recorrência em nefrolitíase. J Bras Urol 1999; 25:475-79.

4. Curhan GC, Willett WC, Rimm EB, Stampfer MJ. A prospective study of dietary calcium and other nutrients and the risk of symptomatic kidney stones. N Engl J Med 1993; 328:833-8.

5. Borghi L, Meschi T, Amato F, Briganti A, Novarini A, Giannini A. Urinary volume, water and recurrences in idiopathic calcium nephrolithiasis: a 5-year randomized prospective study. J Urol 1996; I 55:839-43.

6. Hess B, Mauron H, Ackermann D, Jaeger P. Effects of a 'common sense diet' on urinary composition and supersaturation in patients with idiopathic calcium urolithiasis. Eur Urol 1999; 36: 136-43

7. Hosking DH, Erickson SB, Van den Berg CJ, Wilson DM, Smith LH. The stone clinic effect in patients with idiopathic calcium urolithiasis. J Urol 1983; 130:1115-1 I 18.

8. Martini LA, Heilberg IP, Cuppari L, Medeiros FA, Draibe AS, Ajzen H, et al. Dietary habits of calcium stone formers. Braz J Med Biol Res 1993; 26:805-12.

9. Tiselius HG. Aspects of estimation of the risk of calcium oxalate crystallization in urine. Urol Int 1991; 47:255-9.

10. Tiselius HG, Sandvall K. How are urine composition and stone disease affected by therapeutic measures at an outpatient stone clinic? Eur Urol 1990; 17:206- I2.

II. Almeida WS, Schor N. Epidemiological and metabolic evaluation in renal stone patients living in an specific region of Brazil. Braz J Urol 200।; 27:432-9.
12. Yagisawa T, Chandhoke PS, Fan J. Metabolic risk factors in patients with first-time and recurrent stone formation as determined by comprehensive metabolic evaluation. Urology 1998; 52:750-5.

13. Garzon P, Eisenberg MJ. Variation in the mineral content of commercially available bottled waters: implications for health and disease. Am J Med 1998; 105: 125-30.

14. Schwartz BF, Schenkman NS, Bruce JE, Leslie SW, Stoller ML. Calcium nephrolithiasis: effect of water hardness on urinary electrolytes. Urology 2002; 60:23-7.

15. Agreste SA, Schor N, Heilberg IP. Atualização em nefrologia clínica: papel da constituição físico-química da água potável na litogênese renal. J Bras Nefrol 200 I ; 23:45-8.

16. Giannini S, Nobile M, Sartori L, Carbonare LD, Ciuffreda M, Corrò P, et al. Acute effects of moderate dietary protein restriction in patients with idiopathic hypercalciuria and calcium nephrolithiasis. Am J Clin Nutr 1999; 69:267-7I.

17. Reddy ST, Wang C-Y, Sakhaee K, Brinkley L, Pak CYC. Effect of low-carbohydrate highprotein diets on acid-base balance, stone forming propensity, and calcium metabolism Am J Kidney Dis 2002; 40:265-74.

18. Hess B, Zipperle L, Jaeger P. Citrate and calcium effects on Tamm-Horsfall glycoprotein as a modifier of calcium oxalate crystal aggregation. Am J Physiol 1993; 265:F784F9l.

19. Borghi L, Schianchi T, Meschi T, Guerra A, Allegri F, Maggiore $U$, et al. Comparison of two diets for the prevention of recurrent stones in idiopathic hypercalciuria. N Engl J Med 2002; 346:77-84

Artigo recebido: 27/01/03

Aceito para publicação: 17/02/03 